\title{
REVIEW
}

\section{Application and Progress of Texture Analysis in the Therapeutic Effect Prediction and Prognosis of Neoadjuvant Chemoradiotherapy for Colorectal Cancer}

\author{
Guorong Wang, Zhiwei Wang*, Zhengyu Jin* \\ Department of Radiology, Peking Union Medical College Hospital, \\ Peking Union Medical College \& Chinese Academy of \\ Medical Sciences, Beijing 100730, China
}

Key words: colorectal cancer; texture analysis; neoadjuvant chemoradiotherapy; prognosis

\begin{abstract}
Colorectal cancer is one of the most common malignant tumors, and the morbidity and mortality are increasing gradually over the last years in China. Neoadjuvant chemoradiotherapy (nCRT) is currently applied to the treatment of colorectal cancer patients, and it is helpful to improve the prognosis. The sensitivity of patients to nCRT is different due to individual differences. Predicting the therapeutic effect of nCRT is of great importance for the further treatment methods. Texture analysis, as an image post-processing technique, has been more and more utilized in the field of oncologic imaging. This article reviews the application and progress of texture analysis in the therapeutic effect prediction and prognosis of nCRT for colorectal cancer.
\end{abstract}

OLORECTAL cancer (CRC) is one of the most common malignant tumors. The morbidity and mortality rank the fourth and the second in the world, respectively. Similarly, they are increasing gradually over the last years in China. ${ }^{[1]}$ The radical resection is the primary treatment for CRC patients. However, about $25 \%-30 \%$ of them

Received for publication February 1, 2019. Published online February 25, 2019.

*Corresponding author Zhiwei Wang Tel: 86-10-69155544, E-mail: zhiweiwang1981@sina.com; Zhengyu Jin Tel: 86-1065295441; E-mail: jinzhengyu@163.com

Supported by the National Public Welfare Basic Scientific Research Program of Chinese Academy of Medical Sciences (2018PT32003 and 2017 PT32004). are inoperable as they have been confirmed hepatic metastasis at the initial diagnosis. ${ }^{[2]}$ Neoadjuvant chemoradiotherapy (nCRT) refers to accept the local radiotherapy and systemic chemotherapy before the operation. It can down tumor staging, reduce the recurrence rate and distant metastasis rate of tumors, and help patients improve the prognosis. ${ }^{[3]}$ Currently, nCRT has been mostly applied in patients with CRC. The sensitivity to $\mathrm{nCRT}$ varies because of individual variations and tumor heterogeneity, ${ }^{[4]}$ so it is crucial to predict the therapeutic effect of nCRT for patients for choosing further treatment methods. The imaging examinations have become the vital means to evaluate and predict the therapeutic effect of CRC patients with the development of medical imaging technology. In recent years, the texture analysis technology has 
been applied frequently in oncological imaging. By analyzing the pixel distribution of the lesion displayed on the images, the internal information of the lesion could be quantified, and the heterogeneity and other histopathological characteristics could be reflected more accurately as well. ${ }^{[5-6]}$ This article reviews the application and progress of texture analysis in the therapeutic effect prediction and prognosis of nCRT for CRC patients.

\section{NEOADJUVANT CHEMORADIOTHERAPY}

\section{Importance of neoadjuvant chemoradiotherapy}

nCRT is one of the most significant methods for CRC treatment. Its advantages are mainly manifested as follow: (1) nCRT can shrink the volume of the primary or metastatic tumors, thus reducing staging of the tumors, and increasing resection rate of the lesions.

(2) It has been confirmed that activity of tumor cells would decrease, necrosis and fibrosis of the lesions would appear, and intraoperative dissemination rate and recurrence rate would reduce further after $\mathrm{nCRT}$. $^{[7-8]}$

(3) Tumors would be more sensitive to radiation and relatively high concentration of chemotherapeutic drugs as blood supply and lymphatic vessels of the tumors are not destroyed before operation. ${ }^{[8]}$ (4) nCRT can inhibit or eliminate some potential micrometastases and reduce possibility of long-term metastasis of malignant tumors. (5) Compared with the patients receiving chemoradiotherapy after an operation, most of them are in better physical condition and have a higher tolerance to receive chemoradiotherapy before operation. ${ }^{[8]}(6)$ nCRT can also improve overall survival rate of patients. ${ }^{[9-10]}$

\section{Evaluation criteria for therapeutic effect of neoadjuvant chemoradiotherapy}

Evaluating and predicting therapeutic effect of chemoradiotherapy can reduce overtreatment of patients who are insensitive to the response, avoid the related side effects, and help doctors make more individualized, rigorous, and reasonable treatment plans. Therefore, it is necessary to predict the therapeutic effect of chemoradiotherapy before treatment. Dworak et al. ${ }^{[11]}$ proposed using the tumor regression grading (TRG) to judge the therapeutic effect: 0 , no regression; 1 , mild regression (less than $25 \%$ of the tumor tissue with fibrosis); 2 , moderate regression (26\%-50\% of the tumor tissue with fibrosis); 3 , obvious regression (more than $50 \%$ of the tumor tissue with fibrosis); 4 , com- plete regression (the tumor tissue is not visible and only fibrous tissue is observed). Someone who has scored grade 4 means pathologic complete response ( $P C R$ ) to the chemoradiotherapy, grade 2-3 means partial response (PR), and grade 0-1 means no response (NR).

\section{TEXTURE ANALYSIS TECHNOLOGY}

\section{Basic concepts of texture analysis}

Texture analysis technology is an image post-processing technology, which generates a series of quantitative texture parameters based on the distribution of image pixels or voxels, invisible to the naked eye by using a special filtered algorithm.

Various methods are applied to obtain texture parameters, including the statistical-, transform- and model-based methods. ${ }^{[12]}$ Statistical-based technique has been most commonly applied and extracts specific parameter values by analyzing the distribution and relationships of gray-level values in the image with different methods, mainly including gray-level histogram analysis, gray-level co-occurrence matrix method (GLCM) and neighborhood gray difference matrix method. ${ }^{[13]}$ Gray-level histogram analysis describes the distribution and relationships of gray-level values in the image. It can generate the first-order statistics, such as Mean (mean intensity of a region), standard deviation (SD, variation from mean grey-level value), Skewness (asymmetry of the histogram), Kurtosis (flatness of the histogram), and Entropy (irregularity of gray-level distribution). The second-order texture features are based on GLCM, which describes the distribution relationship between adjacent pixels, and can generate Contrast (local variations and spread of matrix values), Uniformity (uniformity of gray-level distribution), local Entropy (randomness of matrix), and Homogeneity (uniformity of matrix). Higher-order texture features such as Coarseness (the edge density) are based on the neighborhood gray-tone-difference matrices, which mainly describe the spatial distribution among three or more pixels. ${ }^{[13-14]}$ Transform-based methods convert spatial information into Frequency (Fourier, Gabor) and/or Scale (wavelet) information, while model-based texture analysis uses sophisticated mathematical methods such as fractal analysis to represent the texture characteristics of images. ${ }^{[15]}$

\section{The basic operation flow of texture analysis}

The following steps are needed to perform texture 
analysis: (1) obtain the medical images based on the research purpose; (2) delineate the region of interest (ROI) on the selected image; (3) extract texture parameters generated from the texture analysis software from the ROI; (4) analyze data and conclude which texture parameter is significant statistically.

\section{PREDICTION OF THERAPEUTIC EFFECT OF NEOADJUVANT CHEMORADIOTHERAPY IN PATIENTS WITH COLORECTAL CANCER BY TEXTURE ANALYSIS}

\section{Prediction of therapeutic effect of primary lesions}

Texture analysis can reflect the heterogeneity of tumor tissue, ${ }^{[5-6]}$ which is helpful to predict the therapeutic effect of nCRT. The magnetic resonance imaging (MRI) plays an important role in monitoring the therapeutic effect of nCRT in patients with CRC. ${ }^{[16]}$ Although MRI has the characteristics of multi-sequence imaging, most scholars at home and abroad predict the therapeutic effect of nCRT for CRC patients by analyzing the texture features of T2-weighed images (T2WI). De et al. ${ }^{[17-18]}$ found that the Kurtosis value of T2WI in the PCR group was lower than those in the PR group and the NR group of rectal cancer patients before nCRT. It suggested that the Kurtosis value of T2WI before treatment could be used as a predictor of the therapeutic effect of nCRT in rectal cancer patients and help physicians guide the follow-up clinical decision-making as well. Another study ${ }^{[19]}$ showed that the Contrast and Entropy of T2WI before treatment could also predict the therapeutic effect of nCRT for CRC. Liu et al. ${ }^{[20]}$ found that Energy-variance on pre-therapy apparent diffusion coefficient (ADC) mapping was significantly higher in non-responders than that in responders for patients with locally advanced rectal cancer. These studies all indicate that texture analysis based on MRI can be used to predict the effect of nCRT in $\mathrm{CRC}$ patients before treatment, and it is expected to be applied in clinical practice.

In addition to the wide applications in MRI, texture analysis technology has been likewise used in $\mathrm{CT}^{[19,21]}$ and PET-CT ${ }^{[22]}$ images to predict the therapeutic effect of nCRT for CRC. Caruso et al. ${ }^{[19]}$ found that five texture parameters, including the Energy, Contrast and Correlation and so on, could be used as imaging markers to predict the sensitivity of nCRT in CRC patients. Chee et al. ${ }^{[21]}$ confirmed that for patients with locally advanced rectal cancer who responded well to nCRT treatment, the lower the Entropy value was, the higher the uniformity of CT texture images was. When using PET-CT combined with texture parameters to study the effect of nCRT for locally advanced rectal cancer, researchers found that the higher the ratio of the preoperative standard deviation to the mean value was, the better the response to nCRT was. ${ }^{[22]}$

\section{Prediction of therapeutic effect of liver metastases} Liver metastases are one of the main causes of death in patients with CRC. ${ }^{[23]}$ Systemic chemotherapy can reduce liver metastases and prolong survival. ${ }^{[24]}$ Previous studies have suggested that size- or volume-based measurements are limited in evaluating response. ${ }^{[25]}$ Texture analysis technique can quantify the liver parenchyma so that provide more information about chemotherapy-induced changes. ${ }^{[26]}$

In recent years, texture analysis has been confirmed to be helpful in predicting the response to chemotherapy for colorectal liver metastases. Rao et al. ${ }^{[26]}$ found relative differences after chemotherapy in Entropy and Uniformity (without filtration) in CT texture analysis may be better predictors of response to chemotherapy therapeutic effect in patients with colorectal liver metastases. Non-responders showed increased Entropy and decreased Uniformity compared to responders in pre-treatment portal venous phase CT examination. Ahn et al. ${ }^{[27]}$ found that the lower Skewness and SD on liver CT texture images indicated that liver metastases had a better response to chemotherapy. Another study ${ }^{[28]}$ demonstrated that the lower the Entropy value of liver metastasis on CT images was, the better response to chemotherapy was. The lower Skewness, SD, and Entropy indirectly reflect the homogeneity of texture features and also indicate that the lower heterogeneity of tumors is, the higher sensitivity to chemotherapy for candidates is. ${ }^{[27]}$ However, the application of MRI in predicting the chemotherapeutic response of colorectal cancer with liver metastasis is relatively rare. Liang et al. ${ }^{[29]}$ found that the value of Mean from the ADC maps of the responding group were significantly lower than that of the non-responding group in colorectal liver metastases. A latest study ${ }^{[30]}$ exhibited that the higher Entropy, Contrast and the lower Correlation were independently associated with a better chemotherapeutic response in colorectal liver metastases.

\section{Prognosis analysis}

Texture analysis is widely used to predict the survival 
rates of CRC patients. Miles et al. ${ }^{[31]}$ evaluated whether hepatic CT texture and CT perfusion parameters could be related to the survival of patients with CRC. They found that Uniformity of liver texture on portal phase CT images was potentially a superior predictor of survival for patients with CRC than CT perfusion imaging. $\mathrm{Ng}$ et al. ${ }^{[32]}$ analyzed contrast-enhanced CT texture features of CRC lesions and found that Skewness and Kurtosis was associated with 5-year overall survival.

Different imaging techniques combined with texture analysis technology could also estimate the disease-free survival (DFS) of locally advanced rectal cancer. Chee et al. ${ }^{[21]}$ found that homogeneous texture features (higher Uniformity, lower Entropy and lower SD) were connected with higher DFS in patients with locally advanced rectal cancer. Jalil et al. ${ }^{[33]}$ used texture analysis based on MRI to find that Kurtosis was the independent factor to predict DFS for CRC patients. Lovinfosse et al. ${ }^{[34]}$ used ${ }^{18} \mathrm{~F}-\mathrm{FDG}$ PET/CT combined with texture analysis to investigate the prognosis of rectal cancer patients. The results suggested that the Coarseness and Homogeneity were associated with DFS for locally advanced rectal cancer.

All of these studies indicate that it is feasible to assess the prognosis in CRC patients by utilizing texture analysis. We should choose the appropriate examination methods according to the requirements in the clinical work.

\section{Limitations and challenges}

The application of texture analysis technology is becoming more and more extensively, while it still faces challenges and controversies: first of all, most of the studies are retrospective study currently, and the selection bias exists in the enrollment and data acquisition. Second, there is no definite stipulation about optimizing a selection of texture parameters. ${ }^{[35]}$ At last, how to unify the settings and the scanning protocol of various equipment is a problem yet. ${ }^{[36-38]}$

\section{PROSPECT}

In conclusion, texture analysis with its advantages of simplicity and easy operation offers help for predicting the therapeutic effect of nCRT and assessing prognosis for CRC. Given a large amount of data contained in the medical imaging, ${ }^{[39]}$ texture analysis should contribute to complementing and completing the method of extracting the texture features. ${ }^{[40]}$ It is expected to quantify tumor heterogeneity more accurately $^{[41]}$ and make a reasonable explanation of histopathological characteristics represented by different texture parameters under the strict guidance and multimodality imaging, which makes texture parameters as the new imaging biomarker to instruct the clinical diagnosis and treatment.

\section{REFERENCES}

1. Bray F, Ferlay J, Soerjomataram I, et al. Global cancer statistics 2018: GLOBOCAN estimates of incidence and mortality worldwide for 36 cancers in 185 countries. CA Cancer J Clin 2018; 68(6):394-424. doi: 10.3322/caac. 21492.

2. Kemeny $\mathbf{N}$. The management of resectable and unresectable liver metastases from colorectal cancer Curr Opin Oncol 2010; 22(4):364-73. doi: 10.1097/ cCO.0b013e32833a6c8a.

3. Zhang S, Bai W, Tong X, et al. Correlation between tumor microenvironment-associated factors and the efficacy and prognosis of neoadjuvant therapy for rectal cancer. Oncol Lett 2019; 17(1):1062-70. doi: 10.3892/ol.2018.9682.

4. Yang Z, Tang LH, Klimstra DS. Effect of tumor heterogeneity on the assessment of Ki67 labeling index in well-differentiated neuroendocrine tumors metastatic to the liver: implications for prognostic stratification. Am J Surg Pathol 2011; 35(6):853-60. doi: 10.1097/ PAS.0b013e31821a0696.

5. Tsujikawa T, Yamamoto M, Shono K, et al. Assessment of intratumor heterogeneity in mesenchymal uterine tumor by an (18)F-FDG PET/CT texture analysis. Ann Nucl Med 2017; 31(10):752-7. doi: 10.1007/s12149017-1208-x.

6. Kim HS, Kim JH, Yoon YC, et al. Tumor spatial heterogeneity in myxoid-containing soft tissue using texture analysis of diffusion-weighted MRI. PLoS One 2017; 12(7):e0181339. doi: 10.1371/journal.pone.0181339.

7. Van Cutsem E, Cervantes A, Adam R, et al. ESMO consensus guidelines for the management of patients with metastatic colorectal cancer. Ann Oncol 2016; 27(8):1386-422. doi: 10.1093/annonc/mdw235.

8. Li Y, Wang J, Ma X, et al. A review of neoadjuvant chemoradiotherapy for locally advanced rectal cancer. Int J Biol Sci 2016; 12(8):1022-31. doi: 10.7150/ ijbs. 15438.

9. Birbeck KF, Macklin CP, Tiffin NJ, et al. Rates of circumferential resection margin involvement vary be- 
tween surgeons and predict outcomes in rectal cancer surgery. Ann Surg 2002; 235(4):449-57.

10. Rodel C, Grabenbauer GG, Papadopoulos T, et al. Apoptosis as a cellular predictor for histopathologic response to neoadjuvant radiochemotherapy in patients with rectal cancer. Int J Radiat Oncol Biol Phys 2002; 52(2):294-303.

11. Dworak O, Keilholz L, Hoffmann A. Pathological features of rectal cancer after preoperative radiochemotherapy. Int J Colorectal Dis 1997; 12(1):19-23.

12. Davnall F, Yip CS, Ljungqvist G, et al. Assessment of tumor heterogeneity: an emerging imaging tool for clinical practice? Insights Imaging 2012; 3(6):573-89. doi: 10.1007/s13244-012-0196-6.

13. Alobaidli S, McQuaid S, South $C$, et al. The role of texture analysis in imaging as an outcome predictor and potential tool in radiotherapy treatment planning. Br J Radiol 2014; 87(1042):20140369. doi: 10.1259/ bjr.20140369.

14. Scalco E, Rizzo G. Texture analysis of medical images for radiotherapy applications. Br J Radiol 2017; 90(1070):20160642. doi: 10.1259/bjr.20160642.

15. Lubner MG, Smith AD, Sandrasegaran K, et al. CT texture analysis: definitions, applications, biologic correlates, and challenges. Radiographics 2017; 37(5):1483-503. doi: 10.1148/rg.2017170056.

16. Habr-Gama A, Perez RO, Nadalin W, et al. Operative versus nonoperative treatment for stage 0 distal rectal cancer following chemoradiation therapy: longterm results. Ann Surg 2004; 240(4):711-7; discussion 717-8.

17. De Cecco CN, Ganeshan B, Ciolina M, et al. Texture analysis as imaging biomarker of tumoral response to neoadjuvant chemoradiotherapy in rectal cancer patients studied with 3-T magnetic resonance. Invest Radiol 2015; 50(4):239-45. doi: 10.1097/ rli.0000000000000116.

18. De Cecco CN, Ciolina M, Caruso D, et al. Performance of diffusion-weighted imaging, perfusion imaging, and texture analysis in predicting tumoral response to neoadjuvant chemoradiotherapy in rectal cancer patients studied with 3T MR: initial experience. Abdom Radiol (NY) 2016; 41(9):1728-35. doi: 10.1007/ s00261-016-0733-8.

19. Caruso D, Zerunian M, Ciolina M, et al. Haralick's texture features for the prediction of response to therapy in colorectal cancer: a preliminary study. La Radiol Med 2018; 123(3):161-7. doi: 10.1007/s11547-0170833-8.
20. Liu M, Lv H, Liu LH, et al. Locally advanced rectal cancer: predicting non-responders to neoadjuvant chemoradiotherapy using apparent diffusion coefficient textures. Int J Colorectal Dis 2017; 32(7):100912. doi: 10.1007/s00384-017-2835-3.

21. Chee CG, Kim YH, Lee $\mathrm{KH}$, et al. CT texture analysis in patients with locally advanced rectal cancer treated with neoadjuvant chemoradiotherapy: a potential imaging biomarker for treatment response and prognosis. PLoS One 2017; 12(8):e0182883. doi: 10.1371/ journal.pone.0182883.

22. Bundschuh RA, Dinges J, Neumann L, et al. Textural parameters of tumor heterogeneity in (1)(8)F-FDG $\mathrm{PET} / \mathrm{CT}$ for therapy response assessment and prognosis in patients with locally advanced rectal cancer. J Nucl Med 2014; 55(6):891-7. doi: 10.2967/ jnumed.113.127340.

23. Landreau P, Drouillard A, Launoy $G$, et al. Incidence and survival in late liver metastases of colorectal cancer. J Gastroenterol Hepatol 2015; 30(1):82-5. doi: 10.1111/jgh.12685.

24. Chakedis J, Squires $\mathrm{MH}$, Beal EW, et al. Update on current problems in colorectal liver metastasis. Current Probl Surg 2017; 54(11):554-602. doi: 10.1067/ j.cpsurg.2017.10.002.

25. Chung WS, Park MS, Shin SJ, et al. Response evaluation in patients with colorectal liver metastases: RECIST version 1.1 versus modified CT criteria. AJR Am J Roentgenol 2012; 199(4):809-15. doi: 10.2214/ ajr.11.7910.

26. Rao SX, Lambregts DM, Schnerr RS, et al. CT texture analysis in colorectal liver metastases: a better way than size and volume measurements to assess response to chemotherapy? United European Gastroenterol J 2016; 4(2):257-63. doi: $10.1177 / 2050640615601603$

27. Ahn SJ, Kim JH, Park SJ, et al. Prediction of the therapeutic response after FOLFOX and FOLFIRI treatment for patients with liver metastasis from colorectal cancer using computerized CT texture analysis. Eur J Radiol 2016; 85(10):1867-74. doi: 10.1016/ j.ejrad.2016.08.014.

28. Beckers RCJ, Trebeschi S, Maas M, et al. CT texture analysis in colorectal liver metastases and the surrounding liver parenchyma and its potential as an imaging biomarker of disease aggressiveness, response and survival. Eur J Radiol 2018; 102:15-21. doi: 10.1016/j.ejrad.2018.02.031

29. Liang HY, Huang YQ, Yang ZX, et al. Potential of MR 
histogram analyses for prediction of response to chemotherapy in patients with colorectal hepatic metastases. European Radiol 2016; 26(7):2009-18. doi: 10.1007/s00330-015-4043-2.

30. Zhang H, Li W, Hu F, et al. MR texture analysis: potential imaging biomarker for predicting the chemotherapeutic response of patients with colorectal liver metastases. Abdom Radiol (New York) 2019; 44(1):6571. doi: 10.1007/s00261-018-1682-1.

31. Miles KA, Ganeshan B, Griffiths MR, et al. Colorectal cancer: texture analysis of portal phase hepatic CT images as a potential marker of survival. Radiology 2009; 250(2):444-52. doi: 10.1148/radiol.2502071879.

32. Ng F, Ganeshan B, Kozarski R, et al. Assessment of primary colorectal cancer heterogeneity by using whole-tumor texture analysis: contrast-enhanced CT texture as a biomarker of 5-year survival. Radiology 2013; 266(1):177-84. doi: 10.1148/radiol.12120254.

33. Jalil O, Afaq A, Ganeshan B, et al. Magnetic resonance based texture parameters as potential imaging biomarkers for predicting long-term survival in locally advanced rectal cancer treated by chemoradiotherapy. Colorectal Dis 2017; 19(4):349-62. doi: 10.1111/ codi. 13496.

34. Lovinfosse $P$, Polus $M$, Van Daele $D$, et al. FDG PET/ $\mathrm{CT}$ radiomics for predicting the outcome of locally advanced rectal cancer. European J Nucl Med Mol Imaging 2018; 45(3):365-75. doi: 10.1007/s00259-017-
3855-5.

35. Chicklore S, Goh V, Siddique M, et al. Quantifying tumour heterogeneity in 18F-FDG PET/CT imaging by texture analysis. European J Nucl Med Mol Imaging 2013; 40(1):133-40. doi: 10.1007/s00259-012-2247-0.

36. Mackin D, Fave $X$, Zhang $L$, et al. Measuring computed tomography scanner variability of radiomics features. Invest Radiol 2015; 50(11):757-65. doi: 10.1097/ rli.0000000000000180.

37. Fave $X$, Mackin D, Yang J, et al. Can radiomics features be reproducibly measured from CBCT images for patients with non-small cell lung cancer? Med Phys 2015; 42(12):6784-97. doi: 10.1118/1.4934826.

38. Fave $X$, Cook $M$, Frederick $A$, et al. Preliminary investigation into sources of uncertainty in quantitative imaging features. Comput Med Imaging Graph 2015; 44:54-61. doi: 10.1016/j.compmedimag. 2015.04.006.

39. Aerts HJ, Velazquez ER, Leijenaar RT, et al. Decoding tumour phenotype by noninvasive imaging using a quantitative radiomics approach. Nat Commun 2014; 5:4006. doi: $10.1038 /$ ncomms5006

40. Rizzo S, Botta F, Raimondi S, et al. Radiomics: the facts and the challenges of image analysis. European Radiol Exp 2018; 2(1):36. doi: 10.1186/s41747-0180068-z.

41. Summers RM. Texture analysis in radiology: does the emperor have no clothes? Abdom Radiol (New York) 2017; 42(2):342-5. doi: 10.1007/s00261-016-0950-1. 